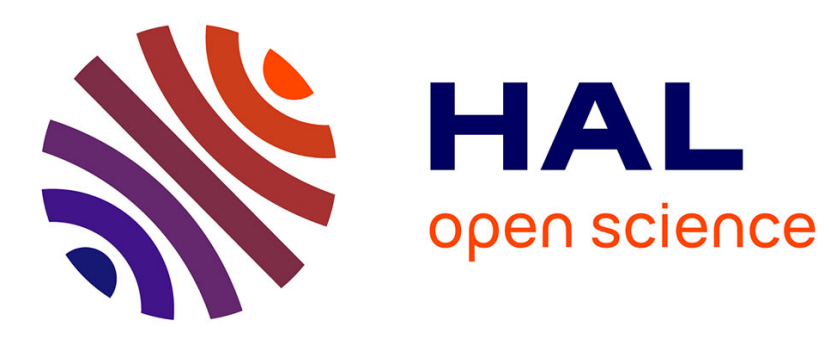

\title{
Les pays de l'Union européenne face aux nouvelles dynamiques des migrations internationales
}

\author{
Franck Bailly, El Mouhoub Mouhoud, Joël Oudinet
}

\section{To cite this version:}

Franck Bailly, El Mouhoub Mouhoud, Joël Oudinet. Les pays de l'Union européenne face aux nouvelles dynamiques des migrations internationales: Ampleur des migrations et caractéristiques des migrants. Revue française des affaires sociales, 2004, 2, pp.33-60. halshs-00487079

\section{HAL Id: halshs-00487079 \\ https://shs.hal.science/halshs-00487079}

Submitted on 31 May 2010

HAL is a multi-disciplinary open access archive for the deposit and dissemination of scientific research documents, whether they are published or not. The documents may come from teaching and research institutions in France or abroad, or from public or private research centers.
L'archive ouverte pluridisciplinaire HAL, est destinée au dépôt et à la diffusion de documents scientifiques de niveau recherche, publiés ou non, émanant des établissements d'enseignement et de recherche français ou étrangers, des laboratoires publics ou privés. 


\title{
Les pays de l'Union européenne face aux nouvelles dynamiques des migrations internationales
}

\author{
Ampleur des migrations et caractéristiques \\ des migrants
}

Franck Bailly, El Mouhoub Mouhoud et Joël Oudinet*

\section{- Introduction}

Une idée souvent répandue est qu'en Europe, à la différence des États-Unis, les migrations sont structurellement et historiquement faibles. Au-delà de la problématique du rôle des migrations de travailleurs dans l'ajustement des marchés du travail des pays de l'Union européenne qui est au centre de l'étude dont est issu ce papier ${ }^{1}$, il apparaissait indispensable de mieux connaître l'ampleur, les caractéristiques et la dynamique des migrations dans l'espace européen, en fonction de critères peu étudiés jusqu'à présent.

En effet, que sait-on vraiment des migrations dans l'espace européen ? Parvient-on à mesurer l'ampleur des flux de migrations pour conclure à leur très grande faiblesse ? Comment appréhender des statistiques basées sur des sources et définitions hétérogènes ? Que savons-nous de leurs caractéristiques en termes d'âge, de qualification, de contrat de travail ? Peu d'études répondent à ces questions de manière systématique et homogène pour les pays européens.

L'un des apports de l'étude dont s'inspire ce papier, est d'avoir exploité de manière détaillée l'enquête sur les forces de travail d'Eurostat (Labor Force Survey-LFS) que nous avons mise en relation avec les données New Cronos (Eurostat) et Sopemi (OCDE) ${ }^{2}$, afin de connaître l'évolution de la population étrangère dans les pays membres de l'Union selon plusieurs critères (nationalité des migrants, pays de naissance, contrats de travail,

\footnotetext{
* Franck Bailly : maître de conférence de l'université de Rouen. Chercheur au Centre d'analyse et de recherche en économie (CARE).

El Mouhoub Mouhoud : professeur à l'université de Paris 13 et directeur du Centre d'économie de Paris Nord (CEPN) CNRS UMR 7115.

Joël Oudinet : maître de conférences à l'université de Paris 13 et chercheur au CEPN.

1 Mouhoud et Oudinet (2003). Cette étude a été effectuée dans le cadre d'un appel d'offre de la MiRe «Circulations migratoires », CEPN, Paris, mai 2003 ; y ont participé : Franck Bailly, Guy Maurau, Jacques Mazier et Sophie Saglio. Les monographies concernant le Portugal et la Turquie ont été réalisées avec Maria Peirera Ramos et Ahmet Insel. Ce rapport est disponible sur simple demande à la MiRe : philippe.bertin@sante.gouv.fr (Tél. : 01405682 34). Voir également Bailly, Mouhoud, Oudinet (2003).

2 Voir annexe pour les sources et les méthodes.
} 
secteur d'activité, niveau de qualification...) et la dynamique des flux migratoires intra et extracommunautaires ${ }^{1}$. Le travail statistique réalisé permet aussi d'appréhender les migrations répétées et d'approcher la question des circulations migratoires dans l'espace européen ${ }^{2}$ qui invite à dépasser la vision souvent trop bilatérale de la migration. La situation de migrations répétées correspond au cas des personnes qui se trouvaient, durant l'année précédente, dans un autre pays que leur pays d'origine et d'accueil au moment de la réponse ${ }^{3}$.

La première section de cet article propose de mesurer l'ampleur des migrations en Europe. Elle montre que, globalement, à l'échelle des quinze pays membres de l'Union européenne, la faiblesse historique des migrations en Europe se confirme lorsque l'on s'intéresse seulement à celles qui proviennent des autres pays membres. Elle souligne également, une forte asymétrie des positions relatives des pays européens en termes d'immigration ${ }^{4}$ : certains ont connu des chocs ponctuels importants (Allemagne), d'autres ont vu leur immigration se stabiliser (France) ou sont passés de la position de pays d'émigration nette à celle de pays d'immigration nette avec une croissance forte de leurs soldes migratoires (Europe du Sud). En outre, la montée de la mobilité des migrants d'origine non communautaire dans l'espace européen semble se dessiner en fonction des marchés du travail européens.

La seconde section présente les caractéristiques des migrants sur les marchés du travail en insistant sur la différence de comportement entre les diplômés et les non diplômés, en fonction de la nature des contrats de travail (temporaire/permanent), de l'importance des marchés internes ou encore du type de secteur d'activité des migrants. Au total, se dessine une sorte de dualisme entre le centre de l'Union européenne et la périphérie à la fois en termes de caractéristiques des migrants et en termes de rôle des pays d'accueil, les pays périphériques jouant, pour les migrants, le rôle de pays relais vers les pays du centre ${ }^{5}$.

\footnotetext{
1 Dans cet article, les termes « migrations intracommunautaires » et « extracommunautaires » désignent respectivement les migrants provenant des quinze pays membres de l'Union européenne et les migrants provenant de pays non membres de l'Union européenne (dont les pays d'Europe centrale et orientale). Par contre, on utilise les termes de « migrants communautaires » et « non communautaires » en fonction de leur nationalité et non plus en fonction de leur pays de provenance. Parfois, le terme d'Européens est utilisé au sens large de l'Union européenne, et désigne la même catégorie que les migrants communautaires.

2 Voir Mouhoud et Oudinet, op cit. chapitres V et VI.

$3 \mathrm{Si}$ l'on tenait compte à la fois des nationaux et des migrants qui changent de pays plusieurs fois dans l'année, le nombre de migrants en situation répétée serait bien entendu plus élevé.

4 Ces asymétries se retrouvent également dans les politiques nationales d'immigration que nous ne traitons pas dans cet article (voir Mouhoud et Oudinet, op cit., chapitre I).

5 Les termes « pays du centre » renvoient aux pays du « noyau dur » de l'Union européenne (France, Allemagne, Royaume-Uni, Pays-Bas, Belgique, Luxembourg). Ce sont aussi des pays traditionnels d'immigration. Les «pays de la périphérie » sont à la fois les pays d'Europe du Sud, l'Irlande et la Finlande.
} 


\section{— Les nouvelles dynamiques migratoires}

L'analyse des migrations dans l'Union européenne permet de dégager deux nouvelles tendances importantes : d'une part, le rôle moteur des nouveaux pays d'accueil dans la relance des migrations et, d'autre part, la complexification des réseaux de migration dont l'apparition du phénomène des migrations répétées constitue l'une des caractéristiques.

\section{Le rôle des nouveaux pays d'accueil}

Les dynamiques migratoires récentes dans l'Union européenne laissent apparaître trois résultats clés :

- Les grands pays d'accueil traditionnels continuent à recevoir la majorité des immigrants de l'Union européenne

Le taux d'immigration moyen (nombre total d'immigrants sur la population totale) de l'ordre de $0,5 \%$ confirme la faiblesse historique des circulations migratoires en Europe ${ }^{1}$. Toutefois, cette faiblesse concerne plus particulièrement les grands pays d'accueil traditionnels (France, Allemagne, Royaume-Uni) qui continuent pourtant à concentrer plus des deux tiers de la population étrangère en Europe. En effet, ces derniers connaissent une stagnation de leur part relative dans l'accueil des étrangers, qui contraste avec l'augmentation des migrations en direction des pays de l'Europe du Sud devenus des pays d'immigration. En outre, la présence des migrants en Europe est également sous-estimée par le critère de la nationalité alors que celui du pays de naissance des migrants laisse apparaittre un chiffre bien plus élevé (23 millions pour l'Europe des Quinze selon le critère du pays de naissance soit $6,3 \%$ de la population totale, contre 17 millions selon le critère de nationalité soit 4,7\% de la population totale en $2000^{2}$ ).

- Si les migrations en Europe ont diminué sur une très longue période, on assiste depuis le milieu des années quatre-vingt à une reprise de ces flux qui s'accélèrent à partir de la fin des années quatre-vingt-dix

La croissance de la population étrangère durant les années quatre-vingt-dix est, en effet, confirmée par l'analyse des données de flux. Depuis 1985, les flux migratoires ont augmenté de plus de $50 \%$ en Europe, avec un taux de croissance annuel moyen de 4,4\% selon les données de l'OCDE ${ }^{3}$. Cette croissance s'accélère encore à la fin des années quatre-vingt-dix comme le montre notre exploitation de l'enquête LFS ${ }^{4}$. Si la croissance des années

1 Données Sopemi-OCDE.

2 Données LFS - nos calculs.

3 Données Sopemi-OCDE.

4 Notre méthode d'exploitation de l'enquête LFS, selon laquelle nous considérons comme migrant toute personne résidant dans un pays où elle ne se trouvait pas l'année précédente, permet en effet de compléter l'analyse des flux d'immigration de l'OCDE. En outre les données LFS, disponibles jusqu'en 2001 pour la majorité des pays, confirment cette accélération de la croissance. 
1989 et 1990 est très largement due au choc de la réunification allemande et de l'ouverture des pays d'Europe centrale et orientale (PECO), l'accélération de la fin des années quatre-vingt-dix dépend plus largement de facteurs économiques, démographiques et sociaux. C'est le cas de la reprise de l'immigration en France et en Allemagne à partir de 1997 (graphique 1), dont les évolutions convergent depuis cette période. Le nombre de personnes résidant à l'extérieur l'année précédente est également proche pour les deux pays (220 000 en France contre 260000 en Allemagne en 2000) ${ }^{1}$.

Graphique 1 : Nombre de migrants (personnes résidant à l'extérieur du pays l'année précédente)

Résidants à l'extérieur du pays l'année précédente

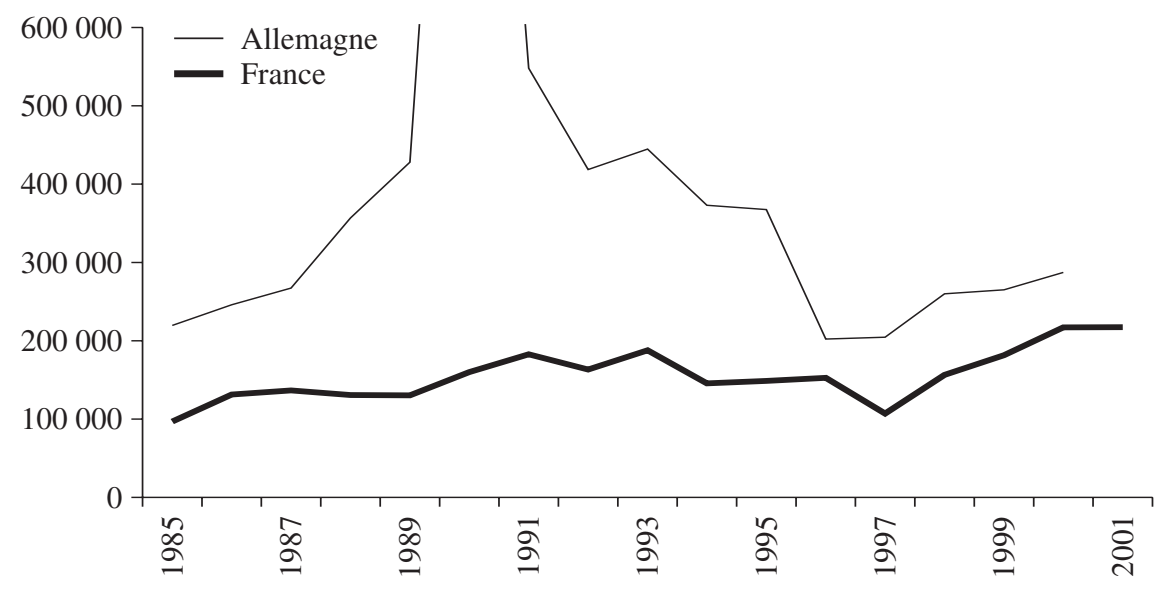

Source : LFS - nos calculs.

- Cette nouvelle croissance de l'immigration en Europe est tirée par la montée de nouveaux pays d'accueil. Ces derniers sont, à la fois, les pays d'Europe du Sud devenus récemment des pays d'immigration nette (Italie, Espagne, Portugal, Grèce) et d'autres pays périphériques comme l'Irlande et la Finlande.

Les pays qui ont connu la plus forte croissance de l'immigration sont l'Espagne, l'Italie et l'Irlande (graphique 2) avec respectivement des taux de croissance annuels moyens de $13,2 \%, 9,1 \%$ et $8,5 \%$ alors que le taux européen est de 4,4 \% sur 1985-1999 2 ${ }^{2}$. Sur les dernières années, l'Espagne et, à un degré moindre, le Portugal deviennent des pays d'accueil de plus en

1 Données LFS - nos calculs.

2 Données Sopemi-OCDE. 
plus attractifs, le nombre de migrants ayant presque doublé au Portugal et triplé en Espagne entre 1999 et $2001^{1}$.

\section{Graphique 2 : Flux d'immigrants dans les pays où la rupture est forte}

\section{Flux total d'immigration}

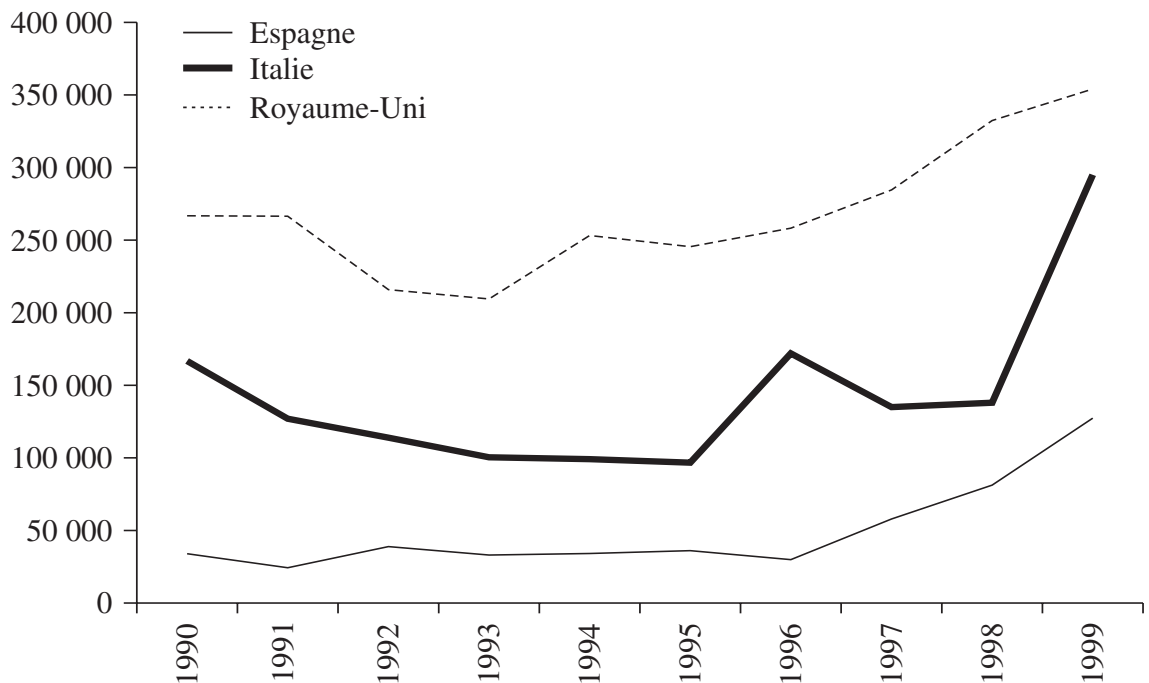

Source : Sopemi-OCDE.

\section{La complexification des réseaux et le décloisonnement des catégories de migrations}

Avec l'achèvement du marché unique européen, les pays membres ont, malgré tout, maintenu des politiques d'immigration différentes voire divergentes, en termes d'ouverture des frontières qui répondent en réalité à des besoins spécifiques. Face à cette diversité des cadres nationaux, les migrants adaptent leur choix de pays de destination en fonction de leurs caractéristiques (le niveau de qualification, l'âge, leur pays d'origine candidat ou non à l'élargissement de l'Union...), ce qui se traduit par une complexification des circulations migratoires dans l'espace européen ${ }^{2}$.

Cette complexification peut être mise en lumière à partir d'une typologie des catégories de migrants (voir tableau 1 et annexe) définie à partir de l'enquête LFS qui croise la nationalité du migrant et son pays de résidence l'année précédente.

1 Données LFS - nos calculs.

2 Cette idée est également nourrie par des études monographiques (Mouhoud et Oudinet, op cit.). 
Tableau 1 : Typologie des catégories de migrants en Europe des Quinze, établie à partir des données d'enquête LFS

\begin{tabular}{|c|c|c|}
\hline \multicolumn{3}{|c|}{ En provenance d'un autre pays de l'Union européenne } \\
\hline $\begin{array}{l}\text { Nationalité Union } \\
\text { européenne } \\
\text { «Européens en mobilité } \\
\text { intracommunautaire » } \\
\text { (1) }\end{array}$ & $\begin{array}{c}\text { Nationaux } \\
\text { « Retours } \\
\text { intracommunautaires » } \\
\text { (3) }\end{array}$ & $\begin{array}{c}\text { Nationalité non Union } \\
\text { européenne } \\
\text { « Non-Européens } \\
\text { en mobilité } \\
\text { intracommunautaire » } \\
\text { (migration répétée) }\end{array}$ \\
\hline \multicolumn{2}{|c|}{ Total européens en mobilité intracommunautaire $(1+3)$} & $(5)$ \\
\hline
\end{tabular}

\begin{tabular}{|c|c|c|}
\hline \multicolumn{2}{|c|}{ En provenance d'un pays extra-Union européenne } \\
\hline $\begin{array}{c}\text { Nationalité } \\
\text { Union européenne }\end{array}$ & Nationaux & $\begin{array}{c}\text { Nationalité non } \\
\text { Union européenne }\end{array}$ \\
$\begin{array}{c}\text { «Européens en mobilité } \\
\text { extracommunautaire } \\
\text { (migration répétée) }\end{array}$ & "Retours \\
$(2)$ & $(4)$ & $\begin{array}{c}\text { Non-Européens } \\
\text { en mobilité } \\
\text { extracommunautaire » }\end{array}$ \\
\hline Total européens en mobilité extracommunautaire (2+4)
\end{tabular}

\begin{tabular}{|c|c|c|}
\hline \multicolumn{3}{|c|}{ Tous pays de départ } \\
\hline $\begin{array}{c}\text { Nationalité } \\
\text { Union européenne } \\
\text { « Migrants européens » } \\
(1)+(2)\end{array}$ & $\begin{array}{l}\text { Nationaux } \\
\text { « Retours » } \\
(3)+(4)\end{array}$ & $\begin{array}{l}\text { Nationalité non } \\
\text { Union européenne } \\
\text { « Migrants } \\
\text { non européens » }\end{array}$ \\
\hline \multicolumn{2}{|c|}{ Total migrants européens au sens large $(1+2+3+4)$} & (J) \\
\hline
\end{tabular}

Note de lecture : le cadre 1 du tableau indique que le migrant a la nationalité d'un des pays de l'Union européenne et qu'il résidait l'année précédente dans un pays de l'Union. Le cadre 2 indique que le migrant a la nationalité d'un des pays de l'Union européenne, mais qu'il résidait l'année précédente dans un pays hors de l'Union.

Trois dimensions caractérisent cette complexification : l'importance effective de la migration intracommunautaire, la diversification des pays d'accueil et la croissance du phénomène des migrations répétées.

\section{Composition et ampleur de la migration intracommunautaire}

Le taux de croissance annuel moyen de l'immigration des communautaires en Europe est de 5,5 \% sur la période 1985-1999, soit légèrement supérieur à la croissance de l'immigration globale qui était de $4,4 \%$. Mais ce sont 
surtout les non-communautaires qui pèsent dans la croissance du nombre total d'immigrants (avec un taux de croissance moyen de $9 \%$ ), alors que le retour des nationaux européens dans leurs pays respectifs est beaucoup plus faible ( $+2 \%$ en moyenne, voir graphique 3 et tableau en annexe 1$)$.

Le tableau 2 fournit le poids relatif de ces différentes catégories sur la période 1995-2000 définies dans la typologie utilisée (tableau 1).

Tableau 2 : Poids relatif des différentes catégories de migrants en fonction de leur nationalité et de leur pays de résidence l'année précédente

\begin{tabular}{|c|c|c|c|c|c|c|c|c|c|}
\hline & (1) & (3) & (5) & $\begin{array}{l}(1)+(3) \\
+(5)\end{array}$ & (2) & (4) & (6) & $\begin{array}{l}(2)+(4) \\
+(6)\end{array}$ & $\begin{array}{c}(2)+(5) / \\
(1)+(2) \\
+(5)+(6)\end{array}$ \\
\hline $\begin{array}{c}\text { Moyenne } \\
\text { sur la période } \\
1995-2000\end{array}$ & $\begin{array}{c}\text { Européens } \\
\text { en mobilité } \\
\text { intra }\end{array} \mid$ & $\begin{array}{c}\text { Retours de } \\
\text { nationaux } \\
\text { intra }\end{array}$ & \begin{tabular}{|c|} 
Non \\
Européens \\
en mobilité \\
intra soit \\
répétée
\end{tabular} & $\begin{array}{c}\text { Mobilité } \\
\text { intra }\end{array}$ & $\begin{array}{c}\text { Européens } \\
\text { en mobilité } \\
\text { extra soit } \\
\text { répétée }\end{array}$ & $\begin{array}{c}\text { Retours de } \\
\text { nationaux } \\
\text { extra }\end{array}$ & $\begin{array}{c}\text { Non } \\
\text { Européens } \\
\text { en mobilité } \\
\text { extra }\end{array}$ & $\begin{array}{c}\text { Mobilité } \\
\text { extra }\end{array}$ & $\begin{array}{c}\text { Mobilité } \\
\text { répétée } \\
\text { (sans } \\
\text { nationaux) }\end{array}$ \\
\hline Autriche & $17 \%$ & $32 \%$ & $5 \%$ & $54 \%$ & $3 \%$ & $16 \%$ & $27 \%$ & $46 \%$ & $15 \%$ \\
\hline Belgique & $41 \%$ & $22 \%$ & $3 \%$ & $66 \%$ & $2 \%$ & $11 \%$ & $21 \%$ & $34 \%$ & $8 \%$ \\
\hline Allemagne & $16 \%$ & $6 \%$ & $1 \%$ & $23 \%$ & $1 \%$ & $29 \%$ & $46 \%$ & $77 \%$ & $2 \%$ \\
\hline Danemark & $4 \%$ & $38 \%$ & $1 \%$ & $43 \%$ & $2 \%$ & $47 \%$ & $7 \%$ & $57 \%$ & $19 \%$ \\
\hline Espagne & $12 \%$ & $21 \%$ & $3 \%$ & $36 \%$ & $3 \%$ & $29 \%$ & $32 \%$ & $64 \%$ & $13 \%$ \\
\hline France & $18 \%$ & $20 \%$ & $2 \%$ & $40 \%$ & $2 \%$ & $28 \%$ & $30 \%$ & $60 \%$ & $7 \%$ \\
\hline Grèce & $4 \%$ & $29 \%$ & $1 \%$ & $34 \%$ & $2 \%$ & $25 \%$ & $39 \%$ & $66 \%$ & $7 \%$ \\
\hline Irlande & $33 \%$ & $36 \%$ & $2 \%$ & $71 \%$ & $1 \%$ & $13 \%$ & $15 \%$ & $29 \%$ & $5 \%$ \\
\hline Luxembourg & $77 \%$ & $5 \%$ & $2 \%$ & $84 \%$ & $5 \%$ & $1 \%$ & $10 \%$ & $16 \%$ & $8 \%$ \\
\hline Pays-Bas & $33 \%$ & $2 \%$ & $1 \%$ & $35 \%$ & $3 \%$ & $12 \%$ & $50 \%$ & $65 \%$ & $4 \%$ \\
\hline Portugal & $6 \%$ & $47 \%$ & $1 \%$ & $54 \%$ & $4 \%$ & $26 \%$ & $16 \%$ & $46 \%$ & $18 \%$ \\
\hline Royaume-Uni & $16 \%$ & $23 \%$ & $4 \%$ & $43 \%$ & $2 \%$ & $27 \%$ & $29 \%$ & $57 \%$ & $11 \%$ \\
\hline $\begin{array}{l}\text { Douze pays } \\
\text { européens }\end{array}$ & $17 \%$ & $18 \%$ & $2 \%$ & $38 \%$ & $2 \%$ & $26 \%$ & $34 \%$ & $62 \%$ & $7 \%$ \\
\hline $\begin{array}{l}\text { Croissance } \\
1999 / 1996 \text { des } \\
\text { douze pays }{ }^{1}\end{array}$ & $19 \%$ & $7 \%$ & $35 \%$ & $14 \%$ & $-11 \%$ & $1 \%$ & $38 \%$ & $21 \%$ & $15 \%$ \\
\hline
\end{tabular}

Note de lecture : $40 \%$ des migrants entrant en France, étaient l'année précédente dans un autre pays européen (mobilité intra) : ces migrants sont soit des nationaux (20\%), soit des immigrants de nationalité européenne (18\%), soit des immigrants non européens (2\%). La décomposition des immigrants arrivant en France en provenance d'un pays extérieur à l'Union européenne (60\%) est identique, soit composée respectivement de $28 \%$ de Français, $2 \%$ d'Européens et $30 \%$ de non Européens. Les données sont arrondies.

${ }^{1}$ Le taux de croissance est celui de la moyenne 1998-1999-2000 comparé à la moyenne 1995-1996-1997, afin de lisser les fluctuations.

Source : LFS - nos calculs. 


\section{Graphiques 3 : Contribution des nationalités au flux total d'immigration}

Contribution au flux d'immigration Allemagne 1985

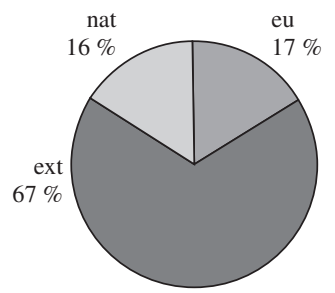

Contribution au flux d'immigration Royaume-Uni 1985

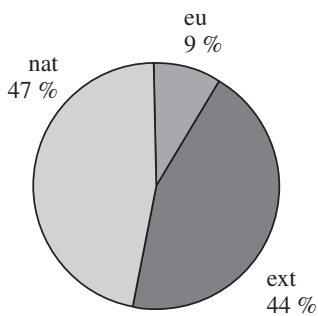

Contribution au flux d'immigration Espagne 1985

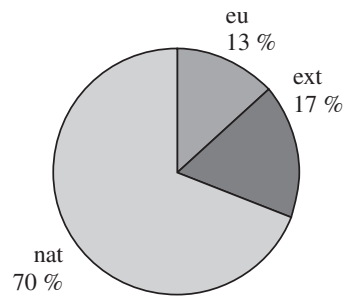

Contribution au flux d'immigration Italie 1985

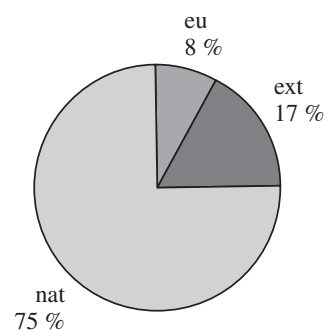

eu : Europe des 15 ; nat : nationaux ; ext : extérieurs.
Contribution au flux d'immigration Allemagne 1999

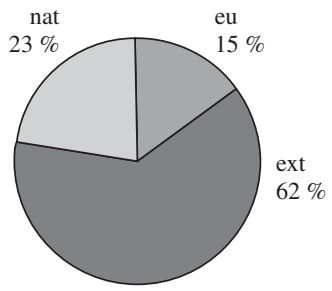

Contribution au flux d'immigration Royaume-Uni 1999

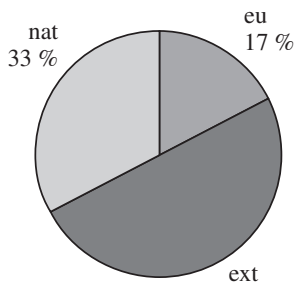

$50 \%$

Contribution au flux d'immigration Espagne 1999

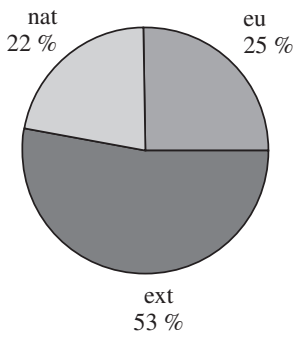

Contribution au flux d'immigration Italie 1999

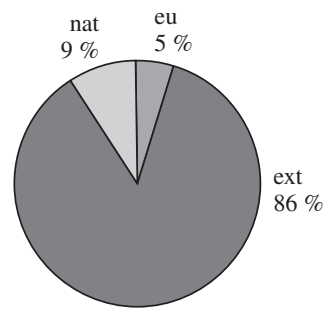

Source : New Cronos. 
L'immigration intracommunautaire est donc plus importante que la seule immigration des personnes de nationalité communautaire puisqu'elle doit prendre en compte les retours de nationaux et les migrations répétées de non communautaires dans l'espace européen (respectivement colonnes 3 et 5). Plus d'un tiers des migrations globales s'effectue au sein de l'Union européenne (38\%), les deux autres tiers de migrants provenant des pays extérieurs aux Quinze de l'Union (62\%). Cette migration est en croissance sur ces trois dernières années $(+14 \%)$ et pourtant un peu plus faible que la migration d'origine extracommunautaire $(+21 \%)$. Il y a une forte diversité entre les pays européens. Certains accueillent majoritairement des migrations intracommunautaires, comme le Luxembourg (84\%), l'Irlande (71 \%), la Belgique (64\%), l'Autriche et le Portugal. Les migrants arrivant en Allemagne viennent par contre majoritairement de l'extérieur de l'Europe (77\%), ainsi que ceux qui immigrent en Grèce (66\%), Pays-Bas $(65 \%)$, Espagne $(65 \%)$ et France $(60 \%)$ (tableau 2$)$.

\section{La diversification des pays d'accueil}

La liste des pays d'immigration ou de destination s'est accrue et les pays d'Europe du Sud sont devenus des pays de transit vers d'autres destinations européennes. Les migrations ne sont donc plus seulement bilatérales.

La France, l'Espagne et le Royaume-Uni ont des caractéristiques migratoires structurelles proches de la moyenne européenne (graphique 4) : les non-Européens en mobilité extracommunautaire (de 30 à $35 \%$ ) et les retours extracommunautaires de nationaux (de 26 à $30 \%$ ) sont dominants. Dans ces trois pays, les Européens et les nationaux en mobilité intracommunautaire représentent environ 16 à $20 \%$ chacun. Les deux dernières catégories sont constituées des Européens et des non-Européens en mobilité répétée (3 à $5 \%$ au total de l'ensemble des migrations).

L'immigration en Allemagne présente par contre des caractéristiques différentes, du fait d'une forte présence $(47 \%)$ de non-Européens en mobilité extracommunautaire (Europe de l'Est, Turquie). Le Luxembourg, la Belgique, l'Irlande et les Pays-Bas accueillent en revanche une forte proportion d'Européens (de 77 à $33 \%$ de leur immigration totale) en mobilité intracommunautaire (colonne $1 \mathrm{du}$ tableau 2).

\section{Les migrations répétées : des logiques contrastées selon la nationalité}

La situation de migration répétée consiste pour un migrant à quitter son pays d'origine (l'Ukraine par exemple) et à se rendre dans un pays européen de résidence (l'Allemagne par exemple : pays cible) après avoir séjourné dans un autre pays européen (par exemple le Portugal : pays relais). La migration est dite répétée dans la mesure où le migrant opère ainsi au moins sa seconde émigration. 


\section{Graphique 4 : Part des migrations intra et extracommunautaires dans les pays de l'Union européenne, moyenne 1995-2000 en pourcentage}

12 pays européens

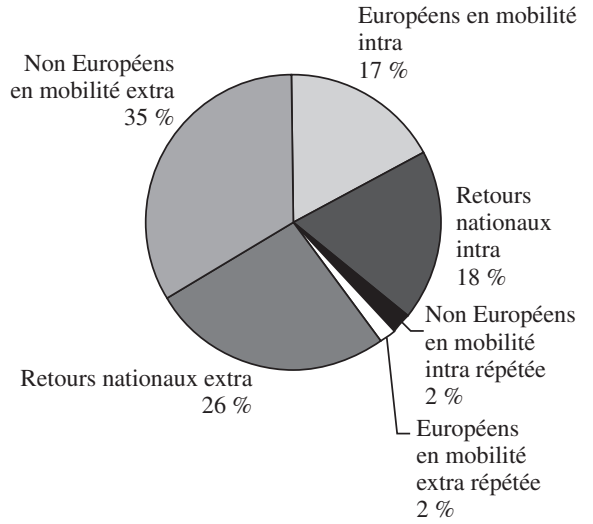

Royaume-Uni

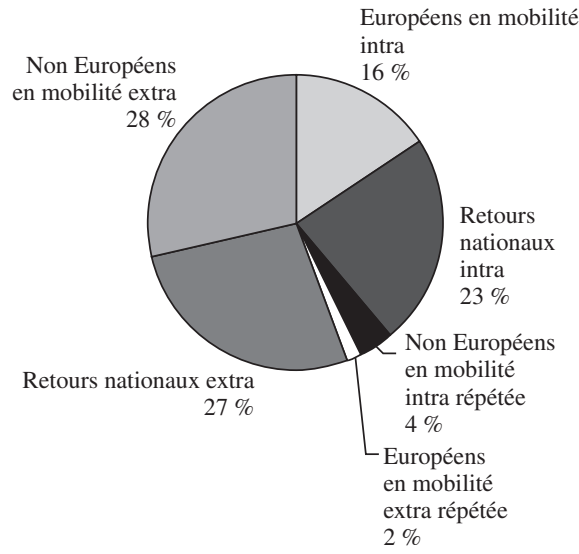

Allemagne

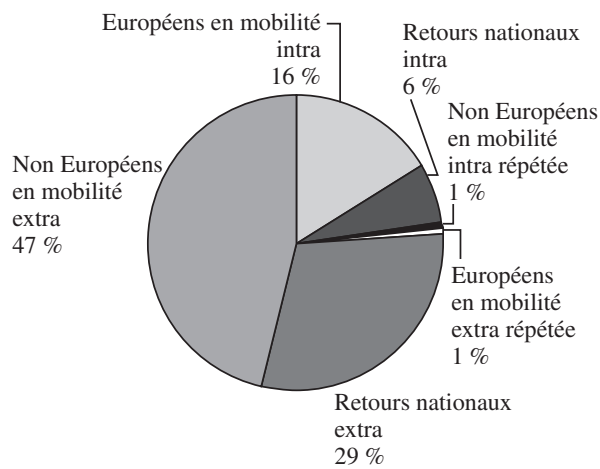

France

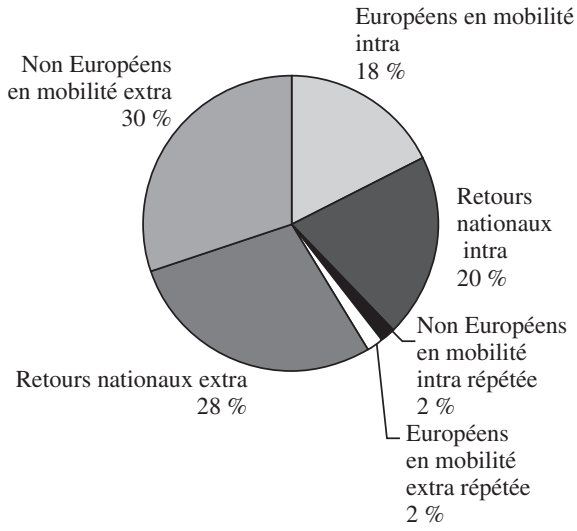

Espagne

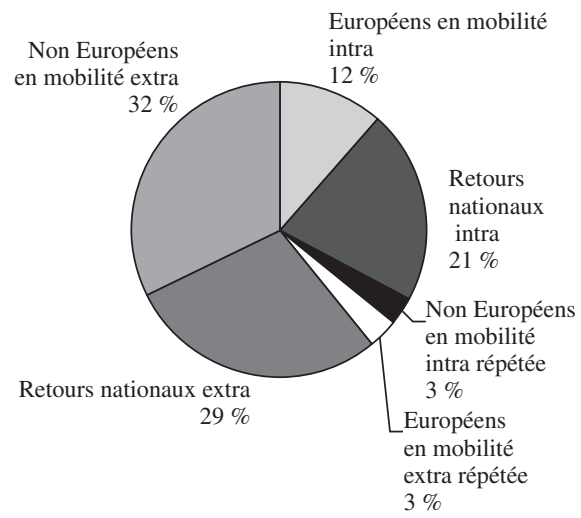

Portugal

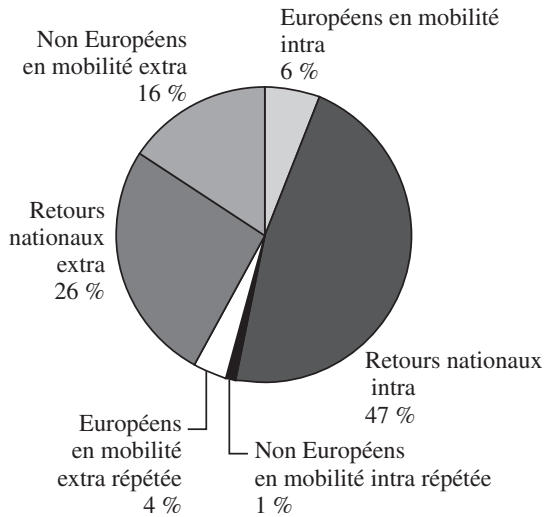

Source : LFS - nos calculs. 
Dans cet exemple, il s'agit d'une migration répétée d'un migrant non communautaire dans l'espace de l'Union européenne (cadran 5 du tableau 1). Un autre exemple de migration répétée serait celui d'un migrant communautaire (par exemple un Français) qui résiderait au moment de l'enquête LFS en Espagne après avoir séjourné dans un autre pays non européen (États-Unis, exemple d'un cadran 2 du tableau 1). L'enquête LFS, ne permet donc de construire et de mesurer que ces deux catégories de migration répétée et sous-estime donc l'ensemble du phénomène ${ }^{1}$. Ce phénomène, certes encore faible, reflète un changement dans la dynamique et les caractéristiques des migrations et préfigure le développement des circulations migratoires.

Sur cette base, on observe que les migrations répétées, de plus en plus fréquentes, concernent plutôt les migrants de nationalité non européenne.

Parmi les migrants européens et non européens, la part de migration répétée est de $7 \%$ pour les pays européens (tableau 2). Le Portugal, l'Espagne, le Danemark et l'Autriche sont particulièrement choisis par les migrants en mobilité répétée, mais pour le Portugal et l'Espagne à un degré moindre, la migration répétée est essentiellement le fait des migrants européens.

L'analyse de ce phénomène révèle une nette différence des comportements des migrants selon leur nationalité d'origine communautaire ou non communautaire.

\section{Les migrants non communautaires : des choix périphériques pour atteindre les pays du centre de l'Union européenne}

Douze à vingt pour cent des immigrants non européens s'installent au Royaume-Uni, Belgique, Autriche et Luxembourg après avoir résidé dans un autre pays de l'Union européenne (graphique 5). Cela signifie sans doute que les migrants les considèrent comme des pays de destination finale ou en tout cas plus définitive puisqu'ils sont déjà passés par un autre pays européen considéré comme « pays relais ». En revanche, les non-Européens qui arrivent en Allemagne, Pays-Bas, France, Portugal et Grèce ne viennent pas ou très peu d'un autre pays européen (moins de $6 \%$ de l'immigration non européenne). Parmi ces pays, on peut distinguer les grands pays d'accueil traditionnels (Allemagne, France et Pays-Bas) qui sont souvent directement ciblés par les migrants à travers les filières classiques, des nouveaux pays d'immigration (Portugal ${ }^{2}$, Grèce) qui jouent un rôle de pays relais.

\footnotetext{
$\overline{1}$ On ne peut pas, en revanche, isoler le migrant communautaire (par exemple un Français) qui résiderait au moment de l'enquête en Espagne après avoir séjourné aussi dans un autre pays de l'Union européenne (par exemple le Royaume-Uni). Ce migrant est en effet comptabilisé dans la même catégorie (cadre 1 des tableaux 1 et 2) qu'un Français qui quitte la France pour l'Espagne où il est comptabilisé au moment de l'Enquête, c'est-à-dire qui n'est pas en migration répétée. De même, un autre cas très spécifique de migration répétée ne peut être décelé, celui du retour de certains nationaux (par exemple un Français revenant en France d'Allemagne après avoir séjourné, précédemment, dans un autre pays, par exemple le Royaume-Uni).

2 Cf. l'article de Maria Pereira Ramos dans le présent numéro.
} 


\section{Graphique 5 : Proportion de non-Européens en migration répétée} arrivant dans un pays de l'Union européenne

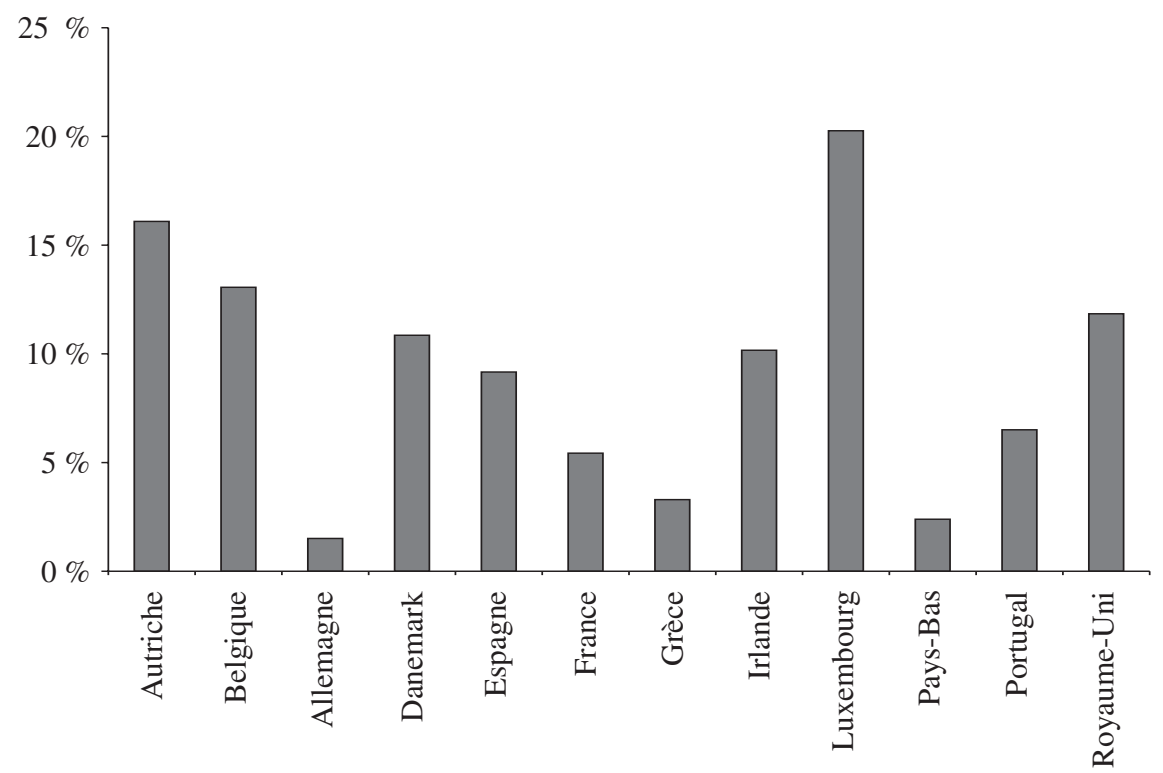

Note de lecture : parmi les non-Européens qui arrivent au Luxembourg (12\% voir colonne $5+6 d u$ tableau 2), $20 \%$ proviennent d'un autre pays européen et sont donc en situation de migration répétée (2\% colonne 5) : $80 \%$ arrivent donc directement de l'extérieur de l'Union européenne (10\% colonne 6).

Source : LFS - nos calculs.

Les caractéristiques des immigrants au Royaume-Uni sont différentes de celles des immigrants s'installant en France ou en Allemagne; plus de $10 \%$ des migrations sont de nature répétée alors que ce type de migration est plus faible dans les autres pays traditionnels d'immigration (3 à $6 \%$ des migrations totales).

Les Allemands et les Néerlandais qui retournent dans leur pays respectif proviennent essentiellement de l'extérieur de l'Union européenne (respectivement 82 et $88 \%$, tableau 2 colonne $4 /(4+3)$ ). Cette situation est très différente de celle des autres pays européens, où la majorité des nationaux reviennent d'un pays de l'Union européenne (près des trois quarts des Irlandais et Luxembourgeois). Les nombreux retours d'Allemands ayant émigré vers les pays de l'Europe de l'Est expliquent ce résultat.

Si l'ampleur de la migration répétée des non-Européens est encore relativement faible, cette catégorie est en forte croissance sur les dernières années (+35\% entre 1996 et 1999) (tableau 2).

Un clivage net se dessine entre les pays qui jouent le rôle de pays cibles et ceux qui jouent le rôle de pays relais. On peut ainsi parler de nouvelles dynamiques migratoires associant pays relais et pays cibles et combinant 
des comportements ou stratégies migratoires différenciés. Le clivage pays cibles/pays relais reflète également des différences dans les politiques d'immigration. Dans les pays cibles, ces politiques sont formellement restrictives en particulier en direction des migrants non diplômés. Dans les pays relais, qui sont à la fois de nouveaux pays d'immigration et des pays périphériques par rapport au noyau dur de l'Union européenne, des stratégies d'ouverture sélective se développent à plus ou moins grande échelle comme dans le cas du Portugal ${ }^{1}$.

Les migrants communautaires : des logiques centrifuges

La situation des Européens en migration répétée est due à un autre aspect de la migration plus généralement liée à la mobilité des personnes diplômées. De 20 à 37 \% des Européens qui migrent au Portugal, en Grèce, en Espagne et au Danemark sont des migrants qui ont déjà migré dans un autre pays de l'Union européenne avant d'arriver dans ces pays (graphique 6). La part d'immigrants européens est assez faible dans ces pays, mais ces migrants sont souvent en situation répétée, au contraire d'autres pays européens. Ces immigrants sont d'ailleurs souvent qualifiés (voir infra).

\section{Graphique 6 : Proportion d'Européens en migration répétée arrivant dans un pays de l’Union européenne}

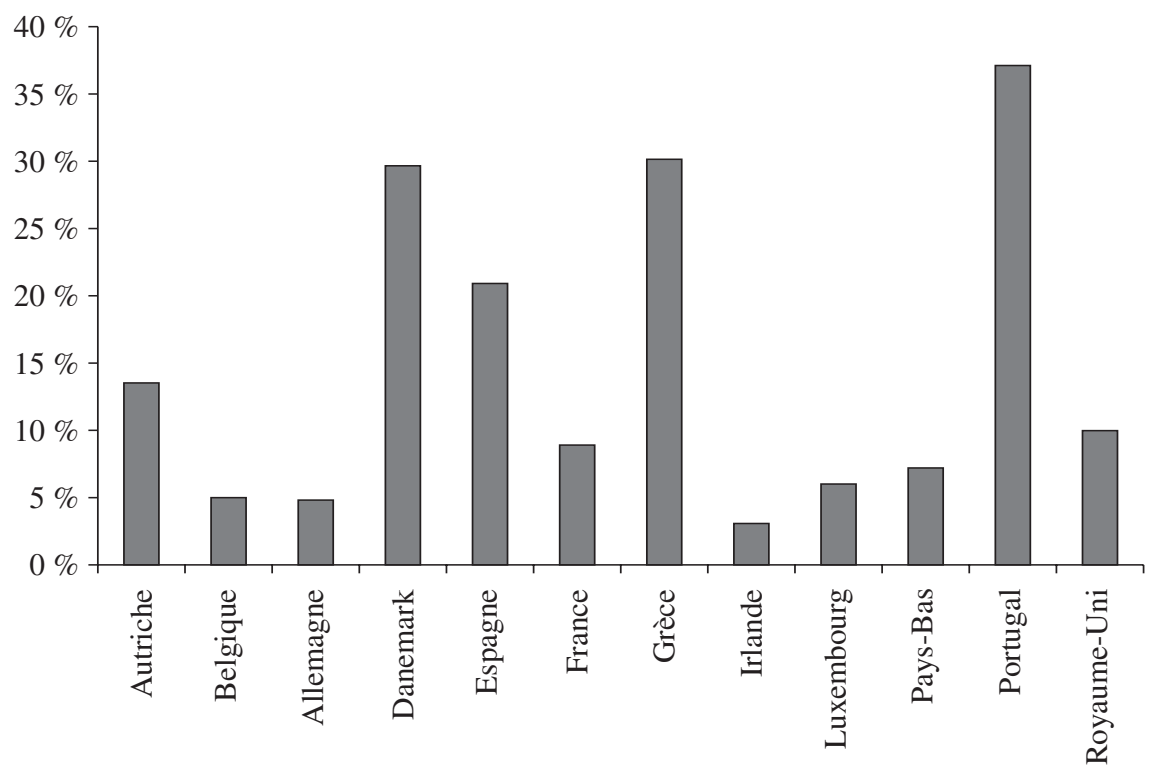

Note de lecture : parmi les Européens qui arrivent en Espagne (14,7\% voir colonne $1+2$ du tableau 2$)$, $21 \%$ proviennent d'un autre pays européen et sont donc en situation de migration répétée (3,1\% colonne 2). $79 \%$, arrivent donc directement de l'extérieur de l'Union européenne (11,6\% colonne 1).

Source : LFS - nos calculs.

1 Voir note précédente. 
Il semblerait donc que les migrants communautaires développent des stratégies de diversification des pays de destination, davantage en direction de la périphérie de l'Union européenne où les opportunités professionnelles sont plus fréquentes.

Cette double tendance concernant les migrations en Europe - rôle croissant des pays du sud de l'Europe dans l'accueil de migrants et le développement des migrations répétées - trouve sans doute une explication dans l'analyse des caractéristiques d'emploi des migrants sur les différents marchés du travail.

\section{- Les migrants sur le marché du travail européen : un dualisme centre-périphérie}

Les travaux économiques consacrés à l'analyse des caractéristiques des emplois occupés par les migrants dans l'Union européenne sont assez peu nombreux. L'exploitation de l'enquête LFS ${ }^{1}$ permet de mieux documenter cet aspect en précisant les stratégies des migrants sur le marché du travail, dans les pays relais et les pays cibles, ainsi que l'influence qu'y jouent les firmes, en particulier multinationales.

Bien sûr, les dispositions en matière de politiques migratoires et de mobilité concernant, par exemple, les conditions d'entrée, la mobilité intrafirme ${ }^{2}$ ou encore les programmes de mobilité étudiante vont influencer les comportements des immigrants et des entreprises. Mais, à l'intérieur de ce cadre, les acteurs ont la possibilité de tisser des stratégies ${ }^{3}$.

Nous proposons d'en rendre compte en organisant cette partie de la façon suivante. Dans un premier temps sont présentées les caractéristiques générales des immigrants qui s'installent en Europe en termes d'âge, de niveaux de qualification ou de lieu de destination. Les conditions d'insertion sur le marché du travail sont ensuite plus précisément abordées. Le type de mobilité, à l'intérieur de marchés internes ou sur des marchés externes, les secteurs d'activité du pays d'accueil dans lesquels les migrants travaillent et, enfin, la nature de leur contrat de travail, permanent ou temporaire seront successivement détaillés.

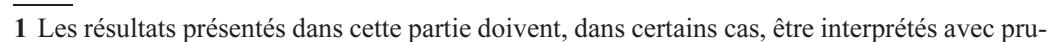
dence au regard de la faible taille des échantillons. Ils présentent néanmoins l'intérêt de dégager des tendances fondées sur des cas précis, à l'aide d'un questionnaire homogène et régulier. 2 Que l'on songe, par exemple, à la directive sur le détachement des salariés.

3 Elles peuvent aller jusqu'au non-respect du droit dans le cas des migrations clandestines. 


\section{Des migrants jeunes et diplômés}

Les migrants qui s'installent dans l'Union européenne sont généralement en âge de travailler. En 1995, $80 \%$ d'entre eux ont entre 15 et 64 ans et $50 \%$ entre 25 et 44 ans ${ }^{1}$. En moyenne, ils sont aussi plus de $20 \%$ à être diplômés de l'enseignement supérieur ${ }^{2}$, avec une tendance à la hausse de cette proportion sur la période.

Les pays d'accueil privilégiés par les migrants diplômés sont l'Allemagne et la France, pour près d'un tiers d'entre eux, et, dans une moindre mesure, le Royaume-Uni en début de période et les Pays-Bas, en fin de période. Le choix de ces pays (France, Allemagne et Royaume-Uni) est plus marqué pour les migrants diplômés d'origines extracommunautaires (tableau 3).

Tableau 3 : Répartition des migrants diplômés en fonction du pays d'accueil

\begin{tabular}{|c|c|c|c|c|c|c|c|c|c|c|c|c|c|}
\hline & AT & BE & DE & FR & $\mathbf{L U}$ & NL & UK & DK & ES & GR & IE & PT & 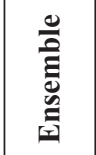 \\
\hline \multicolumn{14}{|c|}{ Migrants communautaires et extracommunautaires } \\
\hline 1995 & $2 \%$ & $2 \%$ & $38 \%$ & $24 \%$ & $1 \%$ & $2 \%$ & $18 \%$ & $2 \%$ & $3 \%$ & $1 \%$ & $6 \%$ & $1 \%$ & $100 \%$ \\
\hline 1999 & $1 \%$ & $2 \%$ & $35 \%$ & $30 \%$ & $1 \%$ & $13 \%$ & $4 \%$ & $1 \%$ & $2 \%$ & $1 \%$ & $7 \%$ & $2 \%$ & $100 \%$ \\
\hline $\begin{array}{l}\text { Moyenne } \\
\text { annuelle } \\
1995-1999\end{array}$ & $2 \%$ & $2 \%$ & $32 \%$ & $31 \%$ & $1 \%$ & $8 \%$ & $7 \%$ & $2 \%$ & $3 \%$ & $1 \%$ & $9 \%$ & $1 \%$ & $100 \%$ \\
\hline \multicolumn{14}{|c|}{ Migrants communautaires } \\
\hline 1995 & $3 \%$ & $4 \%$ & $33 \%$ & $29 \%$ & $2 \%$ & $3 \%$ & $11 \%$ & $2 \%$ & $2 \%$ & $1 \%$ & $10 \%$ & $2 \%$ & $100 \%$ \\
\hline 1999 & $2 \%$ & $5 \%$ & $30 \%$ & $22 \%$ & $2 \%$ & $16 \%$ & $5 \%$ & $1 \%$ & $2 \%$ & $2 \%$ & $12 \%$ & $2 \%$ & $100 \%$ \\
\hline $\begin{array}{l}\text { Moyenne } \\
\text { annuelle } \\
1995-1999\end{array}$ & $3 \%$ & $3 \%$ & $28 \%$ & $28 \%$ & $2 \%$ & $11 \%$ & $5 \%$ & $1 \%$ & $3 \%$ & $1 \%$ & $13 \%$ & $2 \%$ & $100 \%$ \\
\hline \multicolumn{14}{|c|}{ Migrants extracommunautaires } \\
\hline 1995 & $1,0 \%$ & $1 \%$ & $42 \%$ & $21 \%$ & $0,3 \%$ & $1 \%$ & $22 \%$ & $3 \%$ & $4 \%$ & $2 \%$ & $3 \%$ & $0,4 \%$ & $100 \%$ \\
\hline 1999 & $0,1 \%$ & $1 \%$ & $38 \%$ & $36 \%$ & $0,2 \%$ & $11 \%$ & $4 \%$ & $1 \%$ & $3 \%$ & $1 \%$ & $4 \%$ & $1,0 \%$ & $100 \%$ \\
\hline $\begin{array}{l}\text { Moyenne } \\
\text { annuelle } \\
\text { 1995-1999 }\end{array}$ & $1,0 \%$ & $2 \%$ & $35 \%$ & $33 \%$ & $0,3 \%$ & $7 \%$ & $8 \%$ & $2 \%$ & $3 \%$ & $2 \%$ & $5 \%$ & $1,0 \%$ & $100 \%$ \\
\hline
\end{tabular}

Données non disponibles : Finlande, Suède, Italie.

Source : LFS - nos calculs.

1 New Cronos - Eurostat.

2 À partir de l'enquête LFS, on considère ici comme migrants diplômés, les diplômés de l'enseignement supérieur, c'est-à-dire les niveaux V et VI de la classification internationale type de l'éducation. 
Tableau 4 : Part des migrants diplômés dans le total des migrants

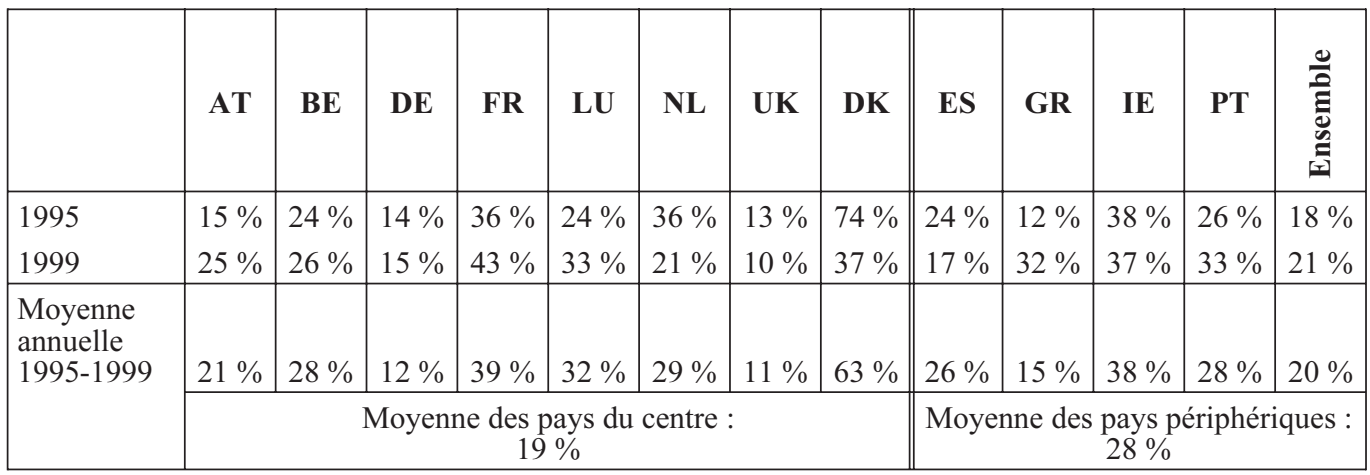

Données non disponibles : Suède, Finlande, Italie.

Note de lecture : en Autriche, en moyenne sur la période 1995-1999, 21 \% des immigrants sont diplomés de l'enseignement supérieur.

Source : LFS - nos calculs.

L'Allemagne et le Royaume-Uni accueillent proportionnellement moins d'immigrants diplômés que la moyenne européenne (tableau 4).

En revanche, en France, la part de diplômés est beaucoup plus élevée (près de $40 \%$ ). On peut proposer une double explication à cette proportion :

- un effet spécifique dû à une politique française très restrictive en matière d'immigration qui s'est traduite par une incitation à l'entrée par des voies alternatives : regroupement familial et transformation des statuts d'étudiants en statut de résident permanent ;

- un effet plus général lié à la montée du niveau de formation et d'éducation dans les pays d'origine des migrants, en particulier en provenance des pays en développement.

La proportion plus élevée de migrants diplômés s'insérant dans les pays périphériques est sans doute à rapprocher de leur rôle de pays relais. Ils sont un passage obligé pour les migrants dont le niveau de qualification est peu reconnaissable directement dans les pays du centre ${ }^{1}$.

Toutefois, il n'y a pas nécessairement d'adéquation entre, d'une part, le niveau de diplôme des migrants et, d'autre part, le type d'emploi occupé, le secteur d'activité et leurs conditions de travail.

\section{Migration et marchés internes}

La mobilité intrafirme (tableau 5), c'est-à-dire qui a lieu sur les marchés internes des firmes multinationales, peut être mesurée, sur la base de

1 Pour une analyse théorique des difficultés de reconnaissance du niveau de qualification sur la migration, voir les travaux de Katz et Stark $(1984,1987)$. 
l'enquête LFS, par le nombre de personnes qui migrent tout en conservant le même employeur.

En 1999, c'est dans les pays du centre que l'on trouve les proportions les plus élevées de migrants qui gardent le même employeur (28\% en moyenne). Dans les pays périphériques, le taux de mobilité intrafirme, est deux fois plus faible que celui des pays du centre (avec une moyenne de $12 \%$ ). Même si l'opposition précédente est moins marquée, une situation similaire se dégage pour les migrants diplômés : ils sont généralement plus nombreux dans les pays du centre à conserver le même employeur que dans les pays périphériques.

Tableau 5 : Part des migrants et des migrants diplômés qui conservent le même employeur dans le total de ceux qui ont un contrat de travail (permanent et temporaire) - année 1999

\begin{tabular}{|c|c|c|c|c|c|c|c|c|c|c|}
\hline $\mathbf{B E}$ & AT & DE & FR & NL & UK & DK & ES & GR & PT & 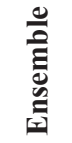 \\
\hline \multicolumn{11}{|c|}{ Ensemble des migrants } \\
\hline $66 \%$ & n.d. & $18 \%$ & $39 \%$ & $45 \%$ & $13 \%$ & $69 \%$ & $6 \%$ & $16 \%$ & $23 \%$ & \multirow[t]{2}{*}{$26 \%$} \\
\hline \multicolumn{7}{|c|}{$\begin{array}{l}\text { Moyenne des pays du centre : } \\
\qquad 28 \%\end{array}$} & \multicolumn{3}{|c|}{$\begin{array}{c}\text { Moyenne des pays } \\
\text { périphériques: } \\
12 \%\end{array}$} & \\
\hline \multicolumn{11}{|c|}{ Migrants diplômés } \\
\hline $63 \%$ & $59 \%$ & $30 \%$ & $42 \%$ & $55 \%$ & $13 \%$ & $88 \%$ & n.d. & $27 \%$ & $40 \%$ & \multirow[t]{2}{*}{$38 \%$} \\
\hline \multicolumn{7}{|c|}{$\begin{array}{l}\text { Moyenne des pays du centre: } \\
\qquad 38 \%\end{array}$} & \multicolumn{3}{|c|}{$\begin{array}{c}\text { Moyenne des pays } \\
\text { périphériques : } \\
33 \%\end{array}$} & \\
\hline
\end{tabular}

n.d. : données non disponibles.

Données non disponibles : Finlande, Suède, Irlande, Italie, Luxembourg.

Note de lecture : en 1999, en Grèce, sur cent migrants qui ont un contrat de travail, seize conservent le même employeur avant et après la migration.

Source : LFS - nos calculs.

Cette opposition traduit probablement un double phénomène. D'une part, il existe une mobilité intrafirme importante à destination des pays périphériques, en provenance d'entreprises implantées dans les pays du centre de l'Union européenne. Ce type de mobilité répondrait, en particulier, à un objectif de transmission de capital humain (Straubhaar et Wolter, 1997 ; Salt, 1997). La part de la mobilité intrafirme, plus élevée pour les diplômés, confirmerait cette hypothèse. D'autre part, il existe une mobilité à destination des pays de la périphérie de l'Union européenne, mais en provenance, cette fois-ci, de pays extérieurs à l'Union européenne, qui passerait davantage par les marchés externes du travail. Plusieurs facteurs peuvent 
expliquer cette situation. En premier lieu, les firmes implantées dans les pays du Sud, extérieurs à l'Europe, et ayant des filiales dans les pays périphériques de l'Union, sont peu nombreuses. Les marchés internes sont donc peu développés. En second lieu, la mobilité externe serait en partie le produit d'un choix opéré par les migrants. Elle augmenterait leur probabilité de trouver un emploi dans le pays d'accueil, souvent dans des conditions de travail très précaires. Enfin, elle permettrait aux employeurs des pays d'accueil de rendre encore plus flexibles leurs politiques de gestion de la main-d'œuvre.

L'analyse des secteurs dans lesquels travaillent les migrants conforte cette interprétation. Ceux qui travaillent dans les pays de la périphérie changent, en effet, plus souvent de secteurs que ceux qui s'installent dans les pays du centre.

\section{Les secteurs d'emplois des migrants dans les pays d'accueil}

En 1999, c'est l'emploi tertiaire qui prédomine dans l'ensemble des pays européens : généralement au moins $60 \%$ des migrants ${ }^{1} \mathrm{~s}$ 'insèrent dans ces activités, ce qui reflète sans doute, une tendance, plus générale, de tertiairisation des économies développées. Cependant, le secteur agricole occupe encore une place non négligeable dans l'emploi des migrants en Grèce $(16 \%)$ et dans une moindre mesure en Espagne $(10 \%)^{2}$.

Un indicateur de stabilité sectorielle du migrant peut être calculé en rapportant, d'une part, le nombre de migrants ayant conservé, dans le pays d'accueil, le même secteur que celui de leur pays d'origine et, d'autre part, le nombre de migrants dans l'ensemble des secteurs du pays d'accueil ${ }^{3}$. Ainsi, en 1999, la part des migrants qui conservent le même secteur d'activité avant et après l'émigration, est plus élevée pour ceux qui trouvent un emploi dans les pays du centre. L'Autriche et le Royaume-Uni, qui se rapprochent des pays périphériques d'Europe du Sud (près de $30 \%$ ), font exception. Dans les pays périphériques, en effet, la part des migrants qui ne changent pas de secteurs lors de leur émigration est plus faible que dans les pays du centre : un sur cinq en Espagne et plus d'un tiers en Grèce (tableau 6). Un tel résultat peut traduire une stratégie d'attente des migrants

1 En France, les résultats de l'Insee (2000) qui s'appuient sur l'enquête sur l'emploi, vont dans le même sens : sur 100 immigrés, 65 sont dans le tertiaire. Est considéré comme immigré pour l'Insee : « une personne résidant en France née à l'étranger et s'étant déclarée de nationalité étrangère ou français par acquisition. Après plusieurs années de séjour en France, un immigré peut demander la nationalité française : devenu français, il fait toujours partie de la population immigrée ".

2 Cf. Mouhoud et Oudinet, op. cit.

3 Avant la migration, les migrants pouvaient être soit sans emploi, soit dans le même secteur que celui dans lequel ils s'insèrent dans le pays de destination, soit dans un secteur différent, soit, enfin, dans un secteur non identifié. 
en vue d'une mobilité sectorielle et géographique future. On retrouve alors l'idée selon laquelle les pays d'Europe du Sud constituent pour les migrants des relais pour atteindre la cible des grands pays d'accueil.

Tableau 6 : Indice de stabilité sectorielle. Part des migrants qui conservent le même secteur avant et après la migration - année 1999

\begin{tabular}{|c|c|c|c|c|c|c|c|c|c|c|c|}
\hline AT & $\mathbf{B E}$ & DK & DE & FR & NL & UK & ES & FI & GR & PT & 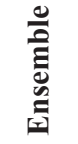 \\
\hline \multicolumn{12}{|c|}{ Ensemble des migrants } \\
\hline $0 \%$ & $88 \%$ & $69 \%$ & $44 \%$ & $49 \%$ & $48 \%$ & $29 \%$ & $22 \%$ & $0 \%$ & $36 \%$ & $26 \%$ & $42 \%$ \\
\hline \multicolumn{7}{|c|}{$\begin{array}{c}\text { Moyenne des pays du centre : } \\
44 \% \\
\end{array}$} & \multicolumn{4}{|c|}{$\begin{array}{c}\text { Moyenne des pays périphériques : } \\
26 \%\end{array}$} & \\
\hline \multicolumn{12}{|c|}{ Migrants diplômés } \\
\hline $0 \%$ & $100 \%$ & $88 \%$ & $50 \%$ & $53 \%$ & $42 \%$ & $59 \%$ & $0 \%$ & $*$ & $62 \%$ & $55 \%$ & \multirow[t]{2}{*}{$51 \%$} \\
\hline \multicolumn{7}{|c|}{$\begin{array}{c}\text { Moyenne des pays du centre: } \\
52 \%\end{array}$} & \multicolumn{4}{|c|}{$\begin{array}{c}\text { Moyenne des pays périphériques : } \\
36 \%\end{array}$} & \\
\hline
\end{tabular}

Données non disponibles : Suède, Irlande, Italie, Luxembourg.

* Pour la Finlande, dans l'enquête LFS on ne trouve aucun migrant diplômé dont le secteur d'emploi est identifié. Dans ce cas, l'indicateur de stabilité sectorielle n'a pas de sens.

Source : LFS - nos calculs.

En ce qui concerne les migrants diplômés, dans la plupart des pays d'accueil, au moins $70 \%$ d'entre eux travaillent dans les activités tertiaires ${ }^{1}$. En outre, les migrants diplômés ont proportionnellement davantage tendance à travailler dans le même secteur d'activité avant et après la migration que les migrants non diplômés (tableau 6). Cela s'explique, sans doute, par la spécialisation professionnelle plus marquée des diplômés et par l'existence de marchés internes et/ou de marchés professionnels plus développés.

Enfin, un dernier critère, la nature juridique du contrat de travail, permet d'apprécier les conditions comparées d'emplois des migrants sur les marchés du travail des pays du centre et de la périphérie de l'Union européenne.

\section{La nature du contrat de travail}

La nature du contrat de travail des migrants, permanent ou temporaire, apporte sur leurs conditions d'insertion dans les pays d'accueil, un autre 
éclairage. Toutefois, ce dernier ne reflète pas seulement les stratégies des migrants ou celles des firmes, mais traduit également les caractéristiques juridiques - notamment en matière de droit du travail - et économiques des marchés de l'emploi des pays de destination. Une manière d'intégrer, en partie au moins, ces spécificités nationales est de comparer la situation des migrants à celle des autres salariés du pays d'accueil. En outre, le caractère temporaire du contrat de travail n'est pas toujours synonyme de précarité. Dans le cas de la mobilité croissante des travailleurs qualifiés et diplômés, le contrat temporaire peut être souvent préféré ou simplement favorisé par les politiques d'immigration des pays d'accueil. Enfin, la précarité peut s'exprimer sous d'autres formes que la nature juridique d'un tel contrat.

Afin d'interpréter de manière précise cet indicateur, on raisonnera d'abord en termes absolus (part des migrants employés sous contrats temporaires sur le total des migrants ayant un contrat) puis en termes relatifs en comparant la situation des migrants à celle de l'ensemble des salariés dans chaque pays d'accueil.

En termes absolus, pour la moyenne de l'Union européenne et sur la période 1995-1999, 37 \% des migrants ont des contrats de travail temporaires ${ }^{1}$ (tableau 7 , colonne (b)). Cela est particulièrement le cas dans les pays périphériques (Espagne, Portugal, Finlande et dans une moindre mesure la Grèce). Dans les pays du centre, les contrats sont davantage des contrats permanents (Autriche, Belgique, Luxembourg) ; font exception les grands pays d'accueil (Royaume-Uni, France et Allemagne) où les travailleurs immigrés sont très largement employés sous contrat à durée déterminée.

Si l'on veut tenir compte des spécificités nationales des marchés du travail dans lesquels s'insèrent les migrants, il convient de caractériser la nature des contrats de l'ensemble des salariés des pays d'accueil. On observe alors que ce sont les employeurs des pays périphériques qui recourent largement à de tels contrats (tableau 4, colonne (d)). C'est le cas en Espagne où cela concerne presque un salarié sur trois, ainsi qu'au Portugal ou en Finlande. À l'inverse, les salariés sont moins concernés par ce type de contrat temporaire dans les pays du centre, particulièrement en Autriche et au Luxembourg.

En termes relatifs, lorsque l'on compare la situation des migrants à celle de l'ensemble des salariés dans chaque pays d'accueil, le rapport entre les deux pourcentages précédents (colonne (e) du tableau 4) systématiquement supérieur à l'unité, révèle que les migrants sont proportionnellement davantage recrutés sur des contrats temporaires que l'ensemble des salariés.

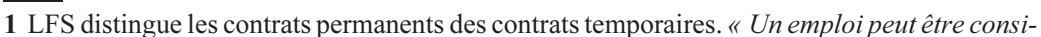
déré comme temporaire s'il est entendu entre l'employeur et le salarié que la fin du contrat est déterminée par des conditions objectives telles qu'une date précise, l'achèvement d'une tâche ou le retour d'un autre salarié qui a été remplacé temporairement ». 
Tableau 7 : Part des contrats temporaires dans le total (contrats temporaires + contrats permanents)

\begin{tabular}{|c|c|c|c|c|c|c|}
\hline & \begin{tabular}{|c|} 
(a) \\
Moyenne \\
annuelle \\
1995-1999 pour \\
les migrants \\
diplômés de \\
l'enseignement \\
supérieur
\end{tabular} & $\begin{array}{c}\text { (b) } \\
\text { Moyenne } \\
\text { annuelle } \\
\text { 1995-1999 } \\
\text { pour les } \\
\text { migrants }\end{array}$ & \begin{tabular}{|c|}
$(\mathbf{c})$ \\
Ensemble de \\
la population \\
salariée \\
diplômée de \\
l'enseignement \\
supérieur - $^{2002^{2}}$ \\
\end{tabular} & $\begin{array}{c}(\mathbf{d}) \\
\text { Ensemble de } \\
\text { la population } \\
\text { salariée - } \\
1999^{1} \\
\end{array}$ & $\begin{array}{c}(\mathrm{e})= \\
\text { (b) } /(\mathrm{d})\end{array}$ & $\begin{array}{c}\text { (f) }= \\
\text { (a) / (c) }\end{array}$ \\
\hline Autriche & $43,0 \%$ & $14,3 \%$ & $4,6 \%$ & $7,5 \%$ & 1,9 & 9,3 \\
\hline Belgique & $20,7 \%$ & $17,8 \%$ & $7,5 \%$ & $10,3 \%$ & 1,7 & 2,8 \\
\hline Luxembourg & $8,6 \%$ & $5,4 \%$ & $2,9 \%$ & $3,4 \%$ & 1,6 & 2,9 \\
\hline Pays-Bas & $17,8 \%$ & $34,1 \%$ & $9,3 \%$ & $12,0 \%$ & 2,8 & 1,9 \\
\hline Allemagne & $39,0 \%$ & $43,2 \%$ & $8,0 \%$ & $13,1 \%$ & 3,3 & 4,9 \\
\hline Finlande & n.d. & $42,0 \%$ & $13,9 \%$ & $18,2 \%$ & 2,3 & n.d. \\
\hline France & $40,5 \%$ & $38,3 \%$ & $13,0 \%$ & $14,0 \%$ & 2,7 & 3,1 \\
\hline Royaume-Uni & $62,0 \%$ & $34,3 \%$ & $7,7 \%$ & $6,8 \%$ & 5,0 & 8,1 \\
\hline Danemark & $38,4 \%$ & $37,4 \%$ & $7,7 \%$ & $10,2 \%$ & 3,7 & 5,0 \\
\hline Espagne & $32,3 \%$ & $49,0 \%$ & $25,7 \%$ & $32,7 \%$ & 1,5 & 1,3 \\
\hline Grèce & $46,7 \%$ & $43,6 \%$ & $8,3 \%$ & $13,0 \%$ & 3,4 & 5,7 \\
\hline Irlande & $20,2 \%$ & $21,1 \%$ & $4,1 \%$ & $9,4 \%$ & 2,2 & 4,9 \\
\hline Portugal & $31,5 \%$ & $56,8 \%$ & $25,6 \%$ & $18,6 \%$ & 3,1 & 1,2 \\
\hline Ensemble & $35,3 \%$ & $37,1 \%$ & & & & \\
\hline
\end{tabular}

1 Pour la Grèce et l'Irlande, il s'agit de l'année 1998.

2 Concerne la population de 15 à 59 ans.

n.d. : données non disponibles.

Données non disponibles : Suède, Italie.

Note de lecture : en Autriche en moyenne sur la période 1995-1999, sur l'ensemble des migrants qui ont un contrat de travail, 14,3\% ont un contrat de travail temporaire. La colonne (e) indique que la part des migrants qui ont des contrats temporaires en Autriche est presque deux fois plus importante (1,9 fois exactement) que la part des salariés qui ont ce type de contrat dans l'ensemble de la population salariée de ce pays.

Source : colonnes (a) et (b) LFS - nos calculs ; colonne (d) Franco (2000) ; colonne (c) : Statistiques sociales européennes (2002).

Ceci s'observe particulièrement dans les pays d'Europe du Sud comme au Portugal et en Grèce où le rapport est supérieur à trois. En Espagne, malgré un marché du travail déjà dominé par des conditions de précarité des salariés, le taux de contrats temporaires des immigrés est supérieur de près de quinze points (différence entre les colonnes (b) et (d)) à celui de l'ensemble des salariés. Au Royaume-Uni, les immigrés sont, pour leur part, cinq fois plus employés sur des contrats temporaires que l'ensemble des salariés. C'est, au contraire, en Autriche, en Belgique et au Luxembourg que la situation des migrants apparait comme la moins défavorable (les ratios de la colonne (e) sont les plus faibles).

Les migrants diplômés sont également plus souvent recrutés sur des contrats temporaires que les salariés diplômés des pays d'accueil : les ratios de la 
colonne (f) sont supérieurs à un. C'est en Espagne et au Portugal que ces ratios sont les plus faibles. Dans la mesure où dans ces deux pays, les marchés du travail sont déjà fortement dominés par la précarité des conditions d'emplois, la situation des migrants diplômés apparaît en termes relatifs un peu moins défavorables ${ }^{1}$.

Toutefois, pour les migrants diplômés comme pour les migrants non diplômés, on peut supposer que cette situation recouvre deux logiques différentes $^{2}$. Dans les pays de la périphérie, les migrants diplômés non européens, pour lesquels les qualifications sont faiblement reconnaissables, acceptent des conditions d'emploi précaires et des emplois moins qualifiés. Ils espèrent ainsi trouver ultérieurement une mobilité professionnelle dans le pays d'accueil ou bien une mobilité géographique et professionnelle dans l'espace européen. En revanche, les pays traditionnels d'accueil, confrontés à des difficultés de recrutement dans certains secteurs, autorisent sous des formes diverses l'entrée temporaire de travailleurs diplômés européens ou non européens, pour lesquels la reconnaissance des qualifications ne pose pas de difficultés.

\section{- Conclusion}

La mobilité des travailleurs au sein de l'Union européenne est en partie alimentée par les migrations extracommunautaires et tend à s'intensifier.

De nouvelles tendances se dessinent :

- d'une part, les grands pays du noyau dur de l'Union européenne, présentant probablement des besoins de main-d'œuvre dans certains secteurs industriels ou des services de haute technologie, font de plus en plus ouvertement appel aux migrants diplômés originaires des pays en développement et des PECO ;

- d'autre part, dans la mesure où l'Union n'est pas une zone homogène, les pays périphériques qui connaissent une divergence structurelle vis-à-vis des pays du centre, tendent à attirer de manière plus ou moins officielle des migrants non communautaires.

Les migrants, de leur côté, développent des stratégies de mobilité géographique et sectorielle successives : ils choisissent l'entrée par les pays périphériques de l'Union européenne qui régularisent rapidement leur situation et acceptent des salaires faibles transitoires ${ }^{3}$ en échange de la possibilité

1 A contrario, toutes choses égales par ailleurs, la situation des migrants apparaît d'autant plus précaire que les employeurs nationaux ont peu recours à des contrats temporaires pour les salariés déjà recrutés dans le pays d'accueil.

2 Cf. Mouhoud et Oudinet, (2003), op. cit., chapitres V et VI.

3 Cette information concernant les salaires des migrants dans les pays d'accueil provient de l'étude de terrain effectuée au Portugal (voir Mouhoud et Oudinet, (2003), chapitre V). 
qui leur est offerte dans un second temps de se procurer « un passeport de mobilité » dans l'espace européen ${ }^{1}$.

En outre, on est conduit à penser que la classification habituelle des migrants (travailleurs, regroupement familial, raisons humanitaires) ne correspond plus à la réalité des flux récents de migrations et aux possibilités exploitées par les migrants de changer de statut : on assisterait ainsi à une sorte de « décloisonnement des catégories de migrants ».

De surcroit, la liste des pays d'immigration ou de destination s'est accrue et les pays d'Europe du Sud sont devenus des pays d'immigration et de transit vers d'autres destinations européennes. Les migrations en situation irrégulière ne concernent pas seulement les travailleurs non qualifiés des pays du tiers-monde, conduits par des filières clandestines opérant dans des secteurs intensifs en travail non qualifié dans les pays d'accueil (bâtiment, textile-habillement, restauration, services...) mais concernent de plus en plus des personnes diplômées en provenance des pays en développement qui passent aussi par des filières plus ou moins formelles et même des emplois non qualifiés en attendant une nouvelle mobilité

Enfin, deux logiques semblent caractériser la situation des migrants en particulier diplômés sur les marchés du travail des pays de l'Union européenne : dans les pays du centre, ils tendent à entrer dans les logiques de flexibilité du marché du travail, tandis que dans les pays périphériques ils subissent de manière plus ou moins provisoire des conditions d'emplois précaires. Cette asymétrie des conditions d'employabilité des migrants entre les pays du centre et ceux de la périphérie constitue l'une des bases importantes du développement récent mais croissant du phénomène des migrations répétées.

1 Il est probable que l'objectif de ces migrants, souvent diplômés ou qualifiés, est d'obtenir, à terme, une adéquation entre leurs qualifications ou leurs diplômes et leurs emplois dans les pays industriels du centre. 


\section{Annexe : Les données sur les migrations : les apports de l'enquête LFS}

Nous croisons différents types de données dans cette étude : des séries directement issues des bases de données relatives aux migrations internationales New Cronos d'Eurostat et Sopemi de l'OCDE, et des données d'enquêtes sur les forces de travail d'Eurostat (LFS).

La collecte des données New Cronos et Sopemi est opérée par Eurostat et l'OCDE à partir de sources nationales dont les critères d'enregistrement et les définitions des migrants sont hétérogènes. Aux différences de critères, vient en effet s'ajouter la diversité des définitions de migrants en fonction de leur durée de résidence.

Les recensements permettent bien d'évaluer précisément, et de façon exhaustive, les effectifs d'étrangers mais leurs périodicités longues et différentes (de cinq à dix ans) empêchent de disposer de données continues.

En revanche, les enquêtes annuelles sur les forces de travail (Labor Force Survey - LFS) nous fournissent ces données chronologiques. Elles présentent aussi le grand avantage d'être homogènes entre les pays tant dans les définitions des variables que dans les critères. C'est pourquoi nous avons choisi d'utiliser essentiellement cette enquête sur les forces de travail réalisée par Eurostat (LFS).

Cette dernière permet d'appréhender la population étrangère selon deux critères : celui de la nationalité au moment de l'enquête et celui du pays de naissance. (On ne peut donc pas repérer la population immigrée sur la base de la définition démographique consistant à comptabiliser les personnes nées à l'étranger et de nationalité étrangère à la naissance). Le croisement de ces deux critères avec celui du pays de résidence l'année précédente permet d'appréhender les flux migratoires intracommunautaires et extracommunautaires.

\section{Une estimation de la population immigrée}

Nous distinguons donc deux types de critère d'utilisation de l'enquête LFS :

- population mesurée selon le critère de nationalité (LFS1) ;

- population mesurée selon le critère du pays de naissance (LFS2).

\section{Selon le critère de la nationalité, trois catégories peuvent être définies}

- Ceux qui possèdent la nationalité du pays d'accueil.

- Ceux qui ne possèdent pas la nationalité du pays d'accueil et qui ont la nationalité d'un pays membre de l'Union européenne. 
- Ceux qui ne possèdent pas la nationalité du pays d'accueil et qui n'ont pas la nationalité d'un pays membre de l'Union européenne.

Ces deux dernières catégories permettent d'appréhender la population totale d'étrangers ${ }^{1}$.

Dans LFS1, on peut distinguer deux types de populations, les étrangers (E) et les nationaux $(\mathrm{N})$ :

Population totale $(\mathrm{PT})=$ étrangers $(\mathrm{E})+$ nationaux $(\mathrm{N})$

Étrangers $(\mathrm{E})=$ étrangers nés à l'étranger $(\mathrm{EE})+$ étrangers nés dans le pays d'accueil (EA)

Nationaux $(\mathrm{N})$ = nationaux nés dans le pays (NA) + naturalisés nés dans le pays $(\mathrm{nA})$ + naturalisés nés à l'étranger $(\mathrm{nE})$ + expatriés rentrés $(\mathrm{EX})$

Conformément à la définition donnée par le Haut Conseil à l'intégration, la population immigrée $(\mathrm{IMM} .=\mathrm{EE}+\mathrm{nE})$ est composée des étrangers nés à l'étranger (EE) et des naturalisés nés à l'étranger $(\mathrm{nE})$.

L'utilisation de la population étrangère en fonction du critère de nationalité mésestime donc le nombre effectif d'immigrés puisque les naturalisés nés à l'étranger (nE) sont exclus de la population étrangère (E). Par contre, les étrangers nés dans le pays d'accueil, ou la seconde génération (EA) sont inclus, ce qui génère un biais non négligeable.

\begin{tabular}{|c|c|c|}
\hline & Immigrés (IMM) & $\begin{array}{c}\text { Nés dans le pays d'accueil } \\
(\mathrm{A})\end{array}$ \\
\hline Étrangers (E) & $\begin{array}{c}\text { Étrangers nés à l'étranger } \\
(\mathrm{EE})\end{array}$ & $\begin{array}{c}\text { Étrangers nés dans le pays } \\
\text { d'accueil (EA) }\end{array}$ \\
\hline Naturalisés & $\begin{array}{c}\text { Naturalisés nés à l'étranger } \\
(\mathrm{nE})\end{array}$ & $\begin{array}{c}\text { Naturalisés nés dans le pays } \\
(\mathrm{nA})\end{array}$ \\
\hline
\end{tabular}

$\mathrm{E}=\mathrm{EE}+\mathrm{EA}$

$\mathrm{IMM} .=\mathrm{EE}+\mathrm{nE}$

Différence entre la population de nationalité étrangère avec la population immigrée

$\mathrm{E}-\mathrm{IMM} .=\mathrm{EA}-\mathrm{nE}$

La population immigrée (IMM) est sous-estimée (surestimée) lorsque la seconde génération (EA) est inférieure (supérieure) aux naturalisés nés à l'étranger $(\mathrm{nE})$. Ainsi, la population de nationalité étrangère $(\mathrm{E})$ en France

\footnotetext{
1 Notons qu'une dernière catégorie est représentée par les individus dont la nationalité n'est pas identifiée au moment de la réponse à l'enquête (non-réponse). Nous avons supposé que cette catégorie puisse être assimilée aux étrangers et plutôt d'origine non communautaire. En effet, après avoir comparé ces données aux autres sources existantes (OCDE-Sopemi, Eurostat-statistiques sociales européennes migrations, New Cronos), on a constaté que la part des étrangers non communautaires dans la population totale était identique aux données de LFS ; cela confirmerait que la catégorie «non-réponse » correspond bien à celle des étrangers non européens.
} 
sous-estime le nombre d'immigrés (IMM) de plus d'un million de personnes selon le dernier recensement de 1999. Ce constat évolue au fil du temps en fonction du droit de la nationalité en vigueur dans chaque pays.

\section{Le critère du lieu de naissance nous donne d'autres indications sur la population immigrée}

Dans LFS2 (la population est mesurée selon le critère du lieu de naissance), on peut distinguer deux types de populations, celles nées à l'étranger (e) et celles nées dans le pays d'accueil (A) :

Population totale $(\mathrm{PT})=$ née à l'étranger $(\mathrm{e})+$ née dans le pays $(\mathrm{A})$

Populations nées à l'étranger (e) = étrangers nés à l'étranger (EE) + naturalisés nés à l'étranger $(\mathrm{nE})+$ expatriés rentrés $(\mathrm{EX})$

Populations nées dans le pays $(\mathrm{n})$ = nationaux nés dans le pays (NA) + naturalisés nés dans le pays (nA) + étrangers nés dans le pays d'accueil (EA) $\mathrm{e}=\mathrm{EE}+\mathrm{nE}+\mathrm{EX}$

$\mathrm{IMM} .=\mathrm{EE}+\mathrm{nE}$

Différence entre la population née à l'étranger avec la population immigrée $\mathrm{e}-\mathrm{IMM} .=\mathrm{EX}$

Mesurer les immigrés $(\mathrm{IMM} .=\mathrm{EE}+\mathrm{nE})$ à partir de ceux qui sont nés à l'étranger (e) est différent : le biais est limité aux expatriés rentrés (EX).

Contrairement à la population de nationalité étrangère (premier critère), la seconde génération (EA) n'est plus comptabilisée. Par contre, sont compris dans cette population née à l'étranger, des personnes ayant la nationalité du pays d'accueil (expatriés rentrés) : par exemple, un Français né en Allemagne et s'installant en France est comptabilisé dans cette population.

\section{Comparaison entre les deux critères}

Dès lors on peut écrire les égalités suivantes :

$\mathrm{E}=\mathrm{EE}+\mathrm{EA}$

$\mathrm{e}=\mathrm{EE}+\mathrm{nE}+\mathrm{EX}$

La différence des deux critères donne $: \mathrm{E}-\mathrm{e}=\mathrm{EA}-(\mathrm{nE}+\mathrm{EX})$

Ainsi, la comparaison entre les deux critères nous permet d'apprécier, pour un moment précis et pour un pays, l'importance des vagues migratoires successives qui ont donné naissance à une seconde génération par rapport au droit de la nationalité en vigueur et à la politique de naturalisations. 
Tableau : Décomposition des flux d'immigrants en fonction de leur nationalité

\begin{tabular}{|c|c|c|c|c|c|c|c|c|}
\hline Pays & & 1985 & 1992 & 1995 & 1999 & $\begin{array}{l}\% \text { annuel } \\
\text { moyen }\end{array}$ & $\begin{array}{c}\text { Coef- } \\
\text { ficient } \\
\text { variable }\end{array}$ & $\begin{array}{l}1999 / \\
1985\end{array}$ \\
\hline \multirow[t]{3}{*}{ Belgique } & UE & 18192 & 27077 & 26551 & 28022 & $3,10 \%$ & 1,9 & $43 \%$ \\
\hline & Non-UE & 19350 & 27973 & 26587 & 29762 & $3,10 \%$ & 3,5 & $43 \%$ \\
\hline & Nationaux & 9500 & 11713 & 9812 & 10682 & $0,80 \%$ & 8,7 & $12 \%$ \\
\hline \multirow[t]{3}{*}{ Danemark } & UE & 3752 & 3776 & 6504 & 8200 & $5,60 \%$ & 1,9 & $78 \%$ \\
\hline & Non-UE & 16449 & 17708 & 32641 & & $1,80 \%$ & 14,3 & $24 \%$ \\
\hline & Nationaux & 16013 & 21893 & 24042 & & $2,60 \%$ & 5,7 & $34 \%$ \\
\hline \multirow[t]{3}{*}{ Allemagne } & UE & 85242 & 120311 & 177200 & 135268 & $3,30 \%$ & 3,4 & $46 \%$ \\
\hline & Non-UE & 344213 & 1091037 & 611000 & 538605 & $3,20 \%$ & 6,2 & $45 \%$ \\
\hline & Nationaux & 82653 & 290850 & 290000 & 200150 & $6,30 \%$ & 7,7 & $88 \%$ \\
\hline \multirow[t]{3}{*}{ Grèce } & UE & 9694 & 3700 & 4748 & 3500 & $-7,30 \%$ & $-3,1$ & $-101 \%$ \\
\hline & Non-UE & 17587 & 11236 & 16111 & & $0,00 \%$ & & $-1 \%$ \\
\hline & Nationaux & 6448 & 17197 & & & $6,80 \%$ & 5,7 & $54 \%$ \\
\hline \multirow[t]{3}{*}{ Espagne } & UE & 2687 & 4484 & 5571 & 32104 & $17,70 \%$ & 2,2 & $248 \%$ \\
\hline & Non-UE & 3489 & 13735 & 13967 & 67018 & $21,10 \%$ & 1,8 & $296 \%$ \\
\hline & Nationaux & 13927 & 20663 & 16554 & 28243 & $5,10 \%$ & 5,4 & $71 \%$ \\
\hline \multirow[t]{2}{*}{ France } & UE & 10181 & 24757 & 8400 & 6500 & $-3,20 \%$ & $-11,1$ & $-45 \%$ \\
\hline & $\begin{array}{l}\text { Non-UE } \\
\text { Nationaux }\end{array}$ & 43619 & 75640 & & 79800 & $5,80 \%$ & 6,2 & $60 \%$ \\
\hline \multirow[t]{3}{*}{ Irlande } & UE & & 10500 & 9000 & 14695 & $8,60 \%$ & 2,1 & $69 \%$ \\
\hline & Non-UE & & 4600 & 4600 & 6905 & $9,60 \%$ & 3,4 & $77 \%$ \\
\hline & Nationaux & & 25600 & 17600 & 25922 & $1,70 \%$ & 9,1 & $13 \%$ \\
\hline \multirow[t]{3}{*}{ Italie } & UE & 6739 & 7209 & 11317 & 15000 & $5,70 \%$ & 3,4 & $32 \%$ \\
\hline & Non-UE & 13765 & 51858 & 56921 & 253000 & $20,80 \%$ & 3 & $228 \%$ \\
\hline & Nationaux & 61679 & 54849 & 28472 & 27000 & $-5,90 \%$ & $-2,7$ & $-76 \%$ \\
\hline \multirow[t]{3}{*}{ Luxembourg } & UE & 4804 & 7077 & 7363 & 8204 & $3,80 \%$ & 1,7 & $31 \%$ \\
\hline & Non-UE & 855 & 2768 & 2227 & 3572 & $10,20 \%$ & 1,7 & $114 \%$ \\
\hline & Nationaux & 923 & 851 & 735 & 1018 & $0,70 \%$ & 19 & $-7 \%$ \\
\hline \multirow[t]{3}{*}{ Pays-Bas } & UE & 13827 & 21446 & 15800 & 20439 & $2,80 \%$ & 3,5 & $39 \%$ \\
\hline & Non-UE & 32339 & 61576 & 53200 & 57926 & $4,20 \%$ & 3 & $58 \%$ \\
\hline & Nationaux & 33196 & 33904 & 29127 & 40786 & $1,50 \%$ & 7,6 & $21 \%$ \\
\hline \multirow[t]{3}{*}{ Autriche } & UE & & & 11300 & 13326 & $4,10 \%$ & 1,1 & \\
\hline & Non-UE & & & & 59053 & $8,50 \%$ & 1,4 & \\
\hline & Nationaux & & & & 14331 & $3,70 \%$ & 0,6 & \\
\hline \multirow[t]{3}{*}{ Portugal } & UE & & 1534 & 2495 & 4568 & $15,10 \%$ & 2,1 & \\
\hline & Non-UE & & 12046 & 2530 & 9908 & $34,10 \%$ & 2,2 & \\
\hline & Nationaux & & & 17000 & 15200 & $-2,80 \%$ & $-7,6$ & \\
\hline \multirow[t]{3}{*}{ Finlande } & UE & 480 & 458 & 900 & 1521 & $8,20 \%$ & 2,3 & $115 \%$ \\
\hline & Non-UE & 2110 & 9892 & 6500 & 6416 & $7,90 \%$ & 3,7 & $111 \%$ \\
\hline & Nationaux & 7875 & 4204 & 4877 & 6807 & $-1,00 \%$ & -14 & $-15 \%$ \\
\hline \multirow[t]{3}{*}{ Suède } & UE & 3469 & 3397 & 7863 & 8836 & $6,70 \%$ & 3,8 & $93 \%$ \\
\hline & Non-UE & 24420 & 36137 & 28216 & 25737 & $0,40 \%$ & 87,4 & $5 \%$ \\
\hline & Nationaux & 5238 & 5814 & 9808 & 15266 & $7,60 \%$ & 1,3 & $107 \%$ \\
\hline \multirow[t]{3}{*}{ Royaume-Uni } & UE & 21300 & 21400 & 45000 & 61943 & $7,60 \%$ & 4,4 & $107 \%$ \\
\hline & Non-UE & 101300 & 95000 & 109200 & 176560 & $4,00 \%$ & 3,9 & $56 \%$ \\
\hline & Nationaux & 109600 & 99500 & 91300 & 115574 & $0,40 \%$ & 40,3 & $5 \%$ \\
\hline \multirow[t]{3}{*}{ UE des Quinze } & UE & & & 342007 & 364125 & $5,50 \%$ & 1,3 & $58 \%$ \\
\hline & Non-UE & & & & 1068058 & $9,00 \%$ & 10,1 & $86 \%$ \\
\hline & Nationaux & & & & & $2,00 \%$ & 6,2 & $26 \%$ \\
\hline
\end{tabular}

Sources : New Cronos et Sopemi. 


\section{Bibliographie}

EUROSTAT, (1998), «Enquête communautaire sur les forces de travail. Méthodes et définitions », Commission européenne.

BAILLY F., MOUHOUD E. M., OUDINET J., (2003), «Union européenne : les nouvelles dynamiques migratoires : relance et complexification », Chroniques internationale de l'IRES, numéro spécial « Mouvements et politiques migratoires, les enjeux sociaux », septembre 2003.

EUROSTAT, (2002), « Statistiques sociales européennes. Résultats de l'enquête sur les forces de travail $2002 »$.

FRANCO A., (2000), « Enquête sur les forces de travail. Principaux résultats 1999 », Statistiques en bref, $\mathrm{n}^{\circ}$ 5/2000.

INSEE PREMIÈRE, (2000), « L’emploi des immigrés en 1999 », nº 717.

KATZ E., STARK O., (1984), « Migration and Asymmetric information : Comment », American Economic Review, $\mathrm{n}^{\circ} 74$.

KATZ E., STARK O., (1987), «International Migration Under Asymetric Information », The Economic Journal, n 97.

MOUHOUD E. M., OUDINET J., (2003), (sous la direction de), Les dynamiques migratoires dans l'Union européenne : ajustements sur les marchés du travail et comparaison Europe-États-Unis, convention CEPN-MiRe, ministère de l'Emploi et des Affaires sociales, 2 tomes.

OCDE, (2001, 2002), Tendances des migrations internationales, Système d'observation permanente des migrations (Sopemi), Paris, OCDE.

SALT J., (1997), « International Movements of the Highly Skilled », OECD Occasional Paper, $\mathrm{n}^{\circ} 3$.

STRAUBHAAR T., WOLTER A., (1997), « Globalisation, Internal Labour Markets and the Migration of the Highly Skilled », Intereconomics, juillet-août. 